\title{
Response to Commentaries on "The Ketamine Model of the Near-Death Experience ..."
}

Karl L. R. Jansen, M.D., Ph.D., M.R.C.Psych. The Maudsley Hospital, London, United Kingdom

\begin{abstract}
The commentators on my paper raised several interesting issues. Set and setting do influence drug effects, but they also influence neardeath experiences (NDEs). Some NDEs are very anxiety-generating, just like some ketamine experiences, though frightening NDEs have been ignored by most researchers. High frequency, compulsive ketamine use is rare. While dimethyltryptamine (DMT) may induce NDEs, this is far from typical, while NDE-like effects are typical of ketamine. Rapidity of onset is not related to the capacity of a drug to induce NDEs. The reality of endopsychosins is doubtful, but the reality of N-methyl-D-aspartate (NMDA) blocking mechanisms is not. NDEs and dream sleep may involve similar mechanisms. Altered states of consciousness do not require a normally functioning brain. Finally, I discuss the possible evolutionary advantage of the NDE mechanism.
\end{abstract}

I would like to commence by expressing my gratitude to the seven commentators for the time and effort taken with these valuable and interesting commentaries.

\section{Response to Rick Strassman}

Rick Strassman began with a discussion of self-experimentation as a valuable and long-standing tradition in medical research. While this is a view that I share, regrettably many others do not, for rea-

Karl L. R. Jansen, M.D., Ph.D., M.R.C.Psych., is a psychiatrist at the Maudsley Hospital. Reprint requests should be addressed to Dr. Jansen at the Maudsley Hospital, Denmark Hill, London SE5 8AZ, United Kingdom. 
sons related both to the overvaluation of statistics, which can conceal as much as they reveal, and to the prevailing sociocultural attitudes towards altered states of consciousness induced by substances. These attitudes are currently reflected in law. Remaining within the law and contemporary ethical guidelines is a requirement that places strict limits upon such research. While it was possible for William James to write of his explorations with consciousness-altering substances in some detail, it is doubtful whether a contemporary professor of psychology at Harvard could do so without censure.

The second major focus is a comparison of the "classical" psychedelic agents, such as lysergic acid diethylamide (LSD), with ketamine. With respect to differences between ketamine-induced states and near-death experiences (NDEs), the central observation is that ketamine is a drug and as such the effects are partially susceptible to set and setting. Ketamine can of course produce states of mind other than NDEs, just as persons who have a myocardial infarction may report altered states of consciousness with a variety of contents, many of which would not be considered to be classical NDEs. The NDE is only one of a spectrum of effects. Scott Rogo has provided an interesting discussion of this issue (Rogo, 1984).

An interesting way to investigate some of these issues would be to administer ketamine and a "classical" psychedelic such as dimethyltryptamine (DMT) to persons who have and have not had NDEs, in double-blind controlled studies. Unfortunately, it is exceptionally difficult to obtain the necessary permissions to do such research in the present climate. Indeed, even the administration of the prescription drug ketamine for nonmedical purposes is a difficult undertaking in some countries, despite its excellent safety record. Accordingly, we are largely reduced to a reliance on the available literature, which leaves little doubt that ketamine does induce a mental state distinctly different from the substances described as classical hallucinogens. Franz Vollenweider has recently described a series of experiments in which subjects were given d-amphetamine, racemic ketamine, S- and R-ketamine, and psilocybin. Positron emission tomography (PET) brain scans were carried out, and altered states of consciousness assessed using the Altered States of Consciousness Questionnaire, the Ego Pathology Inventory, and the inventory of the Association for Methodology and Documentation in Psychiatry (Vollenweider, 1996). While Vollenweider's studies did not specifically address the issue of NDEs, the results tended to support ketamine 
as the drug that reproduces effects most like those of NDEs (see also Vollenweider, 1994).

Strassman argued that one of the differences between NDEs and ketamine anesthesia is that "emergence phenomena" are often unpleasant and there is no desire to repeat them. However, anesthetists have demonstrated that attention to set and setting can make them pleasant. It is also important to recall that some NDEs are not pleasant nor desired to be repeated either, in response to the set and setting in which the NDE occurs. Although some authors have romanticized NDEs, if we listen with an open mind it is quite clear that a substantial fraction are frightening rather than tranquil. This is best seen where persons completely outside the "NDE debate" describe their experiences, as did Marianne Faithfull, former consort of musician Mick Jagger. In her autobiography, Faithfull described several classical but unpleasant NDEs occurring in the context of the end of her relationship with Jagger and the death (possibly by murder) of their friend Brian Jones (Faithfull and Dalton, 1994).

The reason unpleasant NDEs are not often given their due place is that some authors have already made up their minds that NDEs are tranquil by definition, and that if the person describes a frightening experience, he or she cannot have had an NDE. This is a rather limiting perspective, and introduces considerable subjective bias into some books about the NDE. Even if we were to abandon the scientific paradigm completely and accept that NDEs in fact occur during a state of soul transition, our whole experience of the universe is such that we would expect some darkness to accompany the light, one being required to define the other. Even if spiritual explanations are true, it is likely that some NDEs will be perceived as far from pleasant in some persons. Many of the ancient myths and religious ideas have maintained that an experience of a rather unpleasant character was a definite possibility. For example, a substantial fraction of The Tibetan Book of the Dead concerns fearful experiences:

A second sign of Third Bardo existence are experiences of panic, torture and persecution ... [T] [Te form that these torturing demons take will depend on the person's cultural background. (Leary, Metzner and Alpert, 1964, p. 80)

If there were indeed a period of "soul transition," the assertion that this would be universally tranquil is pushing credulity too far. Peter Fenwick addressed the issue of selection bias in his commentary, noting that persons who have had frightening or neutral expe- 
riences are much less likely to want to communicate them than those who have had positive experiences.

Strassman's statement that ketamine is "highly addictive" requires some qualification, and is difficult to reconcile with his statement in the preceding paragraph about the frequently observed desire not to repeat the experience, even allowing for the different contexts of use, anesthetic versus recreational, to which the two statements refer. While there have been famous cases of severely compulsive, excessive ketamine use, these are rare and as such are often the subject of special comment, just as with the classical psychedelic drugs, where continual use is considered to be highly unusual but is nevertheless seen in some persons. The example of John Lilly is a case in point. The account in his autobiography (Lilly, 1978) suggested a highly unusual person who had a need to re-experience the near-death state continually, either through ketamine or by long periods in a flotation tank, in a way that did not actually produce death.

It is also worth noting that Lilly managed to produce a series of NDEs for himself in which real death was imminent by "accidental" actions that an analyst might regard as disguised suicidal acts: for example, going for a bicycle ride down a mountain while under the influence of a phencyclidine (PCP)-like drug, and having a collision with a truck resulting in weeks in the hospital; intravenously injecting a soap bubble together with some LSD in a hotel room, again resulting in hospital admission (the bubble had lodged in his visual artery); passing out in a puddle from which he was saved at the last minute; and so on. It is possible to argue that there are definite similarities between Lilly's seeking of ketamine-induced NDEs, and what appears to have been a compulsive flirtation with real death, resulting in "real" NDEs. Nor is it necessarily true that one action is conscious, the other unconscious. Those persons who do become compulsive ketamine users appear to be as out of control of their actions as a person unconsciously driven to flirt with death. It is of interest to recall the horrific fate of Marcia Moore, the astrologer who co-wrote Journeys Into the Bright World (Moore and Alltounian, 1978), a book about her adventures with ketamine. There are several chilling statements in the book that suggest a person with an unconscious determination to open Bluebeard's cupboard. Moore was found murdered in a forest after the book was completed.

For many people, one ketamine experience, just like one NDE, appears to be more than enough, and the lack of a compulsive desire to repeat the experience is a feature that ketamine and NDEs have 
in common, rather than a point of distinction. It is also true that most persons who have experienced NDEs do not have the means to reproduce them safely, and are thus unable to engage in a process such as that described by Lilly, whether they wished to or not. In general, the use of almost any drug, or indeed almost any human behavior, can become excessive and compulsive in predisposed individuals who are able to practice the indulgence.

Dissociation and dreamlike imagery certainly can occur with classical psychedelic drugs, but it is still clear that in most cases the quality is distinctly different from that produced by the dissociative anesthetics, and that it is ketamine that more reliably reproduces the quality of the NDE, and not psilocybin, DMT, LSD, or mescaline. These latter drugs are not generally regarded as dissociative agents that typically cause NDEs although, as stated in my article, they can certainly do so on occasion. This is because the individual brain can sometimes respond to drugs in markedly idiosyncratic ways, just as immune systems can demonstrate idiosyncratic responses to allergens. There are rare persons who are put to sleep by cocaine, others who are roused by diazepam. Strassman described as outstanding examples of such individual variation two persons who had minimal responses to a high dose of intravenous DMT.

There may be some brains in which LSD and its relatives have neurochemical actions that result, downstream, in N-methyl-D-aspartate (NMDA) receptor blockade, but this is far from the norm. It is possible that there will be some brains where drugs with a primarily serotonergic action such as DMT do result in NDEs. We must always bear in mind the variation in individual response that is possible. The arguments in my hypothesis are for the general case, and certainly allow for exceptions such as DMT-induced NDEs.

The comments concerning rapidity of onset are of interest. Nonmedical ketamine is usually taken orally in the United Kingdom, and usually by mistake, with the users at first believing themselves to have taken another drug, as ketamine is sold fraudulently as the drug methylenedioxymethamphetamine (MDMA), popularly known as Ecstasy. This provides an opportunity to study the effects of the drug in what is almost a double-blind naturalistic study. In brief, the effects of oral ketamine are eventually the same as when it is injected, including the induction of NDEs. Injecting ketamine is relatively rare in the United Kingdom.

PCP also produces an excellent model of the NDE. PCP can result in a state where the consumer believes that he or she is dead for 
several hours. It is a congener of ketamine, both drugs being arylcyclohexylamines, and the ion channel on the NMDA receptor has been named after it. PCP is usually taken orally, which would tend not to support an argument that the rapidity of action is an important feature in whether or not a drug will produce an NDE.

The experiences reported from DMT studies are of interest. The definitive study would administer ketamine to the same persons and examine the differences. It is possible that compulsive use of DMT is rarely seen simply because of the rarity of the drug. Availability has a close relationship to who becomes a compulsive ketamine user and who does not. Indeed, availability is a key issue in any form of addiction. Historically, we have often been proven wrong in predicting that a particular drug is free from the risk of compulsive use as the drug became more available. A recent example of this error was the claim made in this respect for MDMA in the 1980s. My research subjects include a person who injected 250 milligrams of pure MDMA powder four times a day, intravenously, for over six months.

No doubt those involved in the early research upon ketamine and its effects would have been astounded by the level of use engaged in by Lilly, as many persons still are today. It seems reasonable to predict that eventually availability and predisposition to compulsive use will coincide in the case of DMT, if this has not happened already.

\section{Endogenous NDE-Generating Substances}

The brain is a very complex organ, and most mental states have multiple determinants. There are different routes to the same outcome. Thus, for example, a dopamine-releasing agent has a downstream effect upon glutamate release, which is reduced. The central tenet of my hypothesis is that most NDEs result from a reduction in glutamatergic transmission, probably achieved through NMDA receptor blockade in certain pathways in particular parts of the brain. However, the means by which this blockade is brought about is certainly the most speculative part of the hypothesis, and it is highly probable that it could be achieved by several different routes. Certainly the specific compounds labeled "endopsychosins" in the 1980s have little experimental verification of their existence or importance in the human brain. I wished to use the term in a wider sense, to include any endogenous substances that act as NMDA receptor antagonists. As I pointed out in the hypothesis, it may be that an ion 
such as magnesium or zinc is responsible for the NMDA receptor blockade, or some other as yet undiscovered chemical, ion, or indirect process, such as the action of some other neurotransmitter system. The complexity of this receptor macromolecule suggests that the answer to its regulation can never be a simple matter, like the endogenous ligand that was almost immediately discovered for cannabis (THC) receptors.

The case for the existence of DMT in the brain is certainly much stronger that that for the endopsychosins, but this does not dispel the very substantial evidence implicating NMDA receptor blockade in the NDE, however this is achieved. Substances such as DMT may play a role in some part of what will certainly be a multisystem, interactive process. Current evidence, however, does not suggest that DMT itself will be shown to play a central role.

Strassman has made a very valuable contribution to quantifying unusual mental states, and we can only hope that the bodies that control research in these areas will allow this contribution to be more widely applied.

With respect to the religious/philosophical issue, of course the NDE may be studied within these branches of inquiry. However, they are simply not the branches with which my hypothesis is concerned. We would not expect a discussion of neurochemistry from a philosopher or a priest, and why should we? These are simply different paradigms. Why should the person who studies how a radio works also offer a critique upon the music? To fail to do so is not an attempt to rob the music of its beauty, nor does it suggest that we are uncomfortable with music. There is no question that the human mind has a very important, brain-generated capacity for religious experience; there is no ambivalence here of any kind, nor the slightest discomfiture. We are surrounded by religions and their symbols. Of course we routinely use religious and spiritual terms. By the term "nonreligious," I simply meant that I do not adhere to any of the particular systems of beliefs identified with organized religion, and as such the distinctly Dante-esque/Catholic flavor of these NDEs is of interest. Stuart Twemlow and Glen Gabbard might explain this as due to sociocultural forces, and I would agree with them. As Carl Jung pointed out, it is not possible to divorce ourselves from the religious/spiritual aspects of the psyche, which are an integral part of the human package. There can be no doubt that this religious dimension will at some level influence our thoughts and vocabulary to add meaning to our lives. The error occurs when we project this 
inner religious aspect onto the external environment, and really expect to encounter our gods and goddesses in external reality. Many lives have been lost in the name of these projections.

Strassman stated that "the adaptive or biological significance of an endogenous NDE-ogen is difficult to discern." In fact, I discussed the significance of the "endogenous NDE-ogen" in considerable detail in my hypothesis. The major function of such an agent in the glutamate hypothesis of the NDE is that it will protect the brain from excitotoxic cell damage, while generating a mental state that has valuable psychological aspects, such as holding overwhelming anxiety at bay-the usual function of less severe dissociative phenomena in psychology. The psychological advantage may be even greater where the forces of the unconscious are harnessed to give the person a strong message to go back in terms of a mythological drama, and that it is "not their time" - the final expression of the deep drive in the psyche to survive, presented by those parts of the brain still able to produce such a phenomenon. If we consider how much of our brains we can lose in head injuries and yet return to near-normal functioning, and how little electrical activity is recorded during stage 4 sleep and yet the awakened sleeper sometimes records highly significant dream fragments (Kales, 1987; Lask, 1988), it becomes possible to conceptualize the ailing brain screening this final drama, even though there may be very little activity remaining insofar as current instruments are able to detect it. As those who have NDEs do not in fact die, the evolutionary advantage is tremendous.

\section{Response to Peter Fenwick}

Fenwick recorded that he and his wife rejected NDEs that were reported to have occurred while the person was asleep. However, NMDA receptor blockade is also central to the dreaming process, as this also involves sensory deprivation accompanied by an absorbing inner drama. It could be argued that some NDEs result from an activation of the dreaming mechanism, and that alterations in glutamatergic function in certain areas are central to this. Accordingly, there is no need to limit the glutamate hypothesis to a subset of NDEs occurring in catastrophic circumstances. While this subset is useful for highlighting the key issues, the fact remains that glutamate and NMDA receptors play a central role in the brain under all circumstances, and the conditions that activate particular subpro- 
grams are only poorly understood. One of these is the prevention of excitotoxic damage, but there is no reason to suppose that the NDE mechanism would never be activated spontaneously. Indeed, as an expert in epilepsy, Fenwick will be familiar with the sudden onset of major neuroelectrical changes in the brain while persons are "awake and relaxed, when depressed, and in minor infections and in routine anesthesia." When we consider that the brain, and body, can have major seizures "out of the blue," it is not difficult to conceptualize activation of the NDE-generating mechanism in the absence of catastrophe, or indeed any apparent explanation outside of the functioning of the brain itself.

It is of interest to consider also the example of schizophrenia. It is entirely possible to have auditory and visual hallucinations with no apparent precipitating factors whatsoever. An increasingly large number of neuroscientists now believe that the central abnormality in schizophrenia lies in underactivity of the glutamatergic system, with overactivity of dopamine as a variable downstream effect. This is because brain imaging studies show damage in parts of the brain that primarily rely upon glutamate as a neurotransmitter, such as the parahippocampal gyrus. It is of interest to note, in this context, that ketamine increases dopamine but not serotonin concentrations in the blood (Krupitsky, Grinenko, Karandashova, Berkaliev, Moshkov, and Borodkin, 1990), and that ketamine is being explored as a model of schizophrenia (Krystal, Karper, Seibyl, Freeman, Delaney, Bremner, Heninger, Bowers, and Charney, 1994). With respect to the "reality" of ketamine experiences, almost all persons believe their experiences to be real while under the influence of the drug. In one study $30 \%$ of the subjects continued to insist on the reality of their experiences once the drug had worn off.

With respect to the issue of consciousness and a "dysfunctional" brain, some assumptions have been made about the meaning of the terms "clarity," "consciousness," and "dysfunctional," which must be examined. It is useful to consider again the examples of schizophrenia and dreaming. In many cases of schizophrenia, consciousness and clarity appear to be normal, yet there is evidence of brain dysfunction. In dreaming, a very different consciousness and brain organization pertain from that seen in waking, and there is little "clarity," yet we do not say that the brain is dysfunctional, nor do we find it impossible to remember our dreams or ascribe meaning to them. Certainly I would agree with Fenwick that as cerebral function becomes compromised it becomes disorganized, if this term means that it is 
not organized according to its normal waking principles. But there are other ways in which the brain and mind can be organized, and the lesson from mind-altering drugs is that "clarity" in what Lilly referred to as the "social consensus reality" may be very different from "clarity" in an altered state. Indeed, there are those who feel that the true clarity occurs in certain drug-induced states, particularly certain LSD-related states, when the "veil of illusion" is cast aside, and the universe is truly revealed (Grinspoon and Bakalar, 1979). What is confusion to one person may have life-changing meaning to another. We also know how little of our brain is really necessary to maintaining a meaningful form of consciousness, and that a surprisingly large fraction of it can be lost without devastating changes.

It should also be emphasized that the glutamate theory is not a "simple chemical explanation." The brain is clearly extremely complex with a very high level of interactivity and redundancy, and our understanding of its mysteries is in its infancy. The theory does explain how experiences arise in a "dysfunctional" brain, if we consider a brain confused and disorganized by ketamine to be dysfunctional. One doctor's delirium is another's NDE, but most people would agree that falling asleep involves a loss of consciousness, and yet an altered form of consciousness can be very rapidly resynthesized in the form of dreaming. Clearly, there are many forms of consciousness, and the point at which Fenwick and I most clearly diverge in our views is the implication in his argument that there can be only one form of consciousness, in a light-bulb-on/light-bulb-off model, which is based on the medical model of a linear continuum between coma, stupor, clouding, and full consciousness. Certainly a particular level of cortical activity and organization is required to sustain one type of consciousness, the social consensus reality, but this is surely only one type among many. The study of altered states of consciousness is a rich field that includes many states of mind arising from a diversity of cerebral events, many of which are different from the normal one, and some of which may involve organic pathology.

That general terms will no longer suffice in this area is illustrated by recent findings using brain imaging techniques such as positron emission tomography (PET). The injection of ketamine does not simply result in "dysfunction" or general NMDA receptor blockade. Vollenweider (1994) has demonstrated, using PET, that ketamine causes a cortical-subcortical imbalance of sensory information processing. There is a sensory overload of the frontal cortex resulting in a hy- 
permetabolic pattern. This is a specific dysfunction linked to a specific altered state of consciousness. An aim of the study was to explore "the complex inter-relationships between cerebral metabolic changes and psychological changes." It is not unreasonable to assume that there will be specific forms of "dysfunction" responsible for the NDE.

There is no reason why memory of an NDE should present any difficulty for a neurochemical explanation. We remember our dreams without remembering falling asleep, and we remember some parts of altered states produced by drugs but not others. The assumption that we need a normally functioning brain to form a memory would not appear to be supported by most of the evidence. Some persons are very confused on waking, or will even sleepwalk, and yet they can describe their dreams in detail. Even more interesting is the condition known as night terrors (World Health Organization, 1992). This is distinctly different from nightmares. The person wakes from stage 4 sleep (deep sleep), in which brain electrical activity is very simple, with a loud panicky scream and appears confused and disoriented for several minutes. Nevertheless, these persons are able to remember key fragments of dreams that have a particular nature. The most common is a simple dream of entrapment of some kind. These persons explain that they screamed because they developed a sudden selfawareness of having been locked into a place from which there is no escape. Electroencephalography confirms that these are not nightmares, as they do not arise during rapid eye movement sleep, and occur in the first few hours of the night, which is dominated by deep sleep, as distinct from dreams, which are more common in the latter half of the night (Kales, 1987; Lask, 1988). Even more interesting is that this condition, which would appear to be due to an abnormality in the mechanism of sleep, responds to psychotherapy (Kales, 1987; Lask, 1988). Thus no special pleading is required to explain memory in persons who have abnormally functioning brains.

It is also interesting to consider the head injury NDE described by Lilly, who sustained a serious head injury when he collided with the truck, and had an NDE that appeared to last for several weeks. The NDE as described suggests a continuation of the previous experiences with ketamine, rather than a fundamentally different state.

It is possible that in some cases there is little memory for events around the time of a head injury precisely because a dissociative experience has "removed" the person from those events to participate in an inner drama instead. That is, there is a clear memory for an NDE but none for surrounding events, because the person's experi- 
ence was of an NDE. The person with night terrors may remember the dream, but not screaming. All they know of the latter may be the comments of those they live with and a sore throat.

It is noted that medial temporal seizures are associated with fear or extreme fear. However, I did not say that NDEs are medial temporal seizures, nor that ketamine causes medial temporal seizures. What I suggested was that NDEs involve abnormal electrical activity, but this could be of a distinctly different character from these seizures. PET studies with drugs have demonstrated some very complex loops and interaction patterns in the brain. It is also true that some NDEs are associated with panic and negative affect, but this variety less commonly comes to light for the reasons previously discussed.

\section{Response to Igor Kungurtsev}

I had suggested Igor Kungurtsev as a commentator on my paper because he has carried out some very interesting work using ketamine to aid "death-rebirth" psychotherapy. While I hoped that we would learn more of this promising avenue of treatment, his comments here were largely a series of personal beliefs that fall outside of what can be usefully debated in a scientific arena. The real reductionism is seen in the attempt to pull an obscuring, mystical curtain over the study of the NDE, which can advance knowledge no further. The excitement of this historical period lies in the study of the mind/brain interface with the new tools available to us, particularly new brain imaging tools, not in resorting to the myths of-to borrow a term from Kungurtsev- "archaic" religions that have not advanced our understanding for several thousand years. The value of neuroscientific studies that constantly produce many new facts, ideas, and medicines to improve the human condition would appear now to exceed that of ancient, stagnant speculations about reincarnation, which generate nothing new. All they have to say is that NDEs are evidence for the continuation of consciousness after the death of the body. True or false, there is no more that they can add.

The ketamine that Kungurtsev used in his transpersonal investigations was developed by scientists who believed in the laws of physics. While we owe much to real physics, metaphysics kept the world in suspended animation throughout a very long period of history now referred to as the Dark Ages. It was the proponents of everlasting life, chief amongst whom was the Pope, who would not look through 
Galileo's telescope. In some respects, the new real-time neuroimaging techniques represent the new telescope, even more threatening to our sense of self-importance, meaning, and desire for control over biological reality than ever. Unfortunately, our psychological maturation as a species still lags far behind technological advance, as was the case in Galileo's day-less than an eye-blink away in evolutionary terms.

As Kungurtsev did not wish to accept the term "death" for the final end, which term would he like to use for the final end of life? This is a simply a matter of linguistics, and has no relevance to any matters of a philosophical, religious, or spiritual nature, unless, of course, he wishes to argue that no life ever ends and thus we do not need a word for its end. We will certainly need some sort of word for the permanent failure to function of the body, if we are to communicate meaningfully with each other. Dictionaries represent the consensus view on the meaning of a word. Those who do not accept the consensus will find their communication with the rest of us increasingly impaired.

I would like to end these comments with a quote from Albert Einstein, a scientist who has had a far greater impact to date on our lives than have parapsychologists:

The fairest thing we can experience is the mysterious . ... It was the experience of mystery-even if mixed with fear-that engendered religion. A knowledge of the existence of something we cannot penetrate, of the manifestations of the profoundest reason and the most radiant beauty, which are only accessible to our reason in their most elementary forms - it is this knowledge and this emotion that constitute the truly religious attitude; in this sense, and in this alone, I am a deeply religious man. I cannot conceive of a God who has a will of the type of which we are conscious in ourselves. An individual who should survive his physical death is also beyond my comprehension, nor do I wish it otherwise; such notions are for the fears or absurd egoism of feeble souls. Enough for me the inkling of the marvelous structure of reality. $(1935$, p. 5$)$

\section{Response to Melvin Morse}

Melvin Morse again raised the issue of the evolutionary advantage of the NDE. This matter was dealt with both in my original paper and above. In brief, the NDE results from protective forces against brain damage and also has psychological advantages in preventing 
the organism from being overwhelmed with anxiety. The psychological advantage may be even greater where the forces of the unconscious are harnessed to give the person a strong message to go back, and that it is not their time. As those who have NDEs do not in fact die, the evolutionary advantage could be tremendous. Unfortunately, we do not know if persons who do actually die have an NDE.

The literature describing the effects of ketamine in the same terms as the NDE is perhaps not as weak as Morse described it. In fact, ketamine has been administered many times in clinical anesthetic studies. I did not cite this large quantity of anesthetic literature, primarily for practical reasons: my paper already had many references. I wished to present a hypothesis for discussion, not a definitive review of the effects of ketamine, as I cited several excellent reviews to which the interested reader may turn. Those accounts of the effects of ketamine I have presented here are amongst the most interesting and graphic, but there are many more scientific accounts in peer-reviewed journals. A computer search on "ketamine" will produce a vast quantity of formal research, much of it by anesthetists publishing methods of preventing the patient from having an NDE. Such a search would probably not reveal some of the more intriguing references cited here, which is an even better reason for bringing them to the light of day. Lester Grinspoon and James Bakalar (1979) commenced their book with a quote from Alfred North Whitehead, which could be considered here, and which is certainly believed by the near-death researchers Morse mentioned, such as Raymond Moody and Kenneth Ring:

The rejection of any source of evidence is always treason to that ultimate rationalism which urges forward science and philosophy alike. (p. vii)

With respect to quoting speculations by Ronald Siegel, this was done more to credit Siegel's contribution to aspects of the hypothesis than as evidence, which would indeed be inappropriate.

\section{Response to Stuart Twemlow and Glen Gabbard}

Twemlow and Gabbard offered valuable insights upon the psychodynamic and sociocultural aspects of the NDE, areas of considerable interest and, where the psychodynamic aspects are concerned, possible future therapeutic promise. 
On the philosophical/religious issue again, I do regret making these comments about mind/brain dualism. The response has fully confirmed that the paradigms should not be confused. Most of the commentators have spent much space on these few, preliminary paragraphs, and very little upon the lengthy neuroscientific discussion, which is where the new ideas, possibility of experiments, and new avenues in psychotherapy lie. Clearly, I should not have discussed the paradigms on the same stage at all, not even to the extent of a few paragraphs, as this has served as a massive distraction at the expense of a proper consideration of the neuroscience itself.

That more mundane accounts could logically be expected from a state with a neurobiological origin is true because there is then no absolute requirement for an experience with religious/spiritual import, as there would be if the NDE had a religious/spiritual explanation. However, I had no intention to exclude explanations based on intrapsychic and sociocultural factors by my use of the term "neurobiological." I completely adhere to the view of Twemlow and Gabbard that intrapsychic factors and cultural factors are important in generating NDEs.

\section{Response to Antonio Bianchi}

Antonio Bianchi has provided an interesting commentary and discussion, with much valuable information and new ideas. However, the ketamine doses he quoted were very much lower than those generally used to alter consciousness. His text stated that 1 to 2 milligrams intravenously or 3 to 5 milligrams intramuscularly of ketamine induces a trance-like state. These figures are not in accordance with those in the published literature. In most cases, it is unlikely that less than 20 milligrams intravenously of the ParkeDavis product Ketalar would produce a trance-like state. Bianchi cited the book by Moore and Alltounian (1978). These authors clearly stated their doses as 50-75 milligrams of ketamine intramuscularly.

That the effects of ketamine, like all drugs, can be influenced to some extent by set and setting is a matter that has already been discussed in relation to Strassman's comments. This does not mean that different drugs will have the same effect in identical set and settings, however. LSD and ketamine are both likely to produce unpleasant results when given in hospital environments without adequate preparation of administrator and subject. However, the quality 
of that unpleasantness will be very different, reflecting the individual character of the two drugs. The same can be said of DMT.

With respect to current interest in the use of ibogaine to treat addictions, ketamine has also been used for this purpose with an excellent success rate, and a much superior safety record (Krupitsky, Grinenko, Berkaliev, Paley, Tetrov, Mushkov, and Borodkin, 1992; Krupitsky, Grinenko, Karandashova, Berkaliev, Moshkov, and Borodkin, 1990; Krupitsky, Paley, Berkaliev, Ivanov, Dubrovina, Kohanzarova, Dunaevsky, Rhizankova, and Grinenko, 1993). The use of ketamine in the treatment of alcoholism is an area of great future promise.

That ibogaine binds to NMDA receptors is also of great interest from an ethnobotanical perspective. All of the powerful mind-altering substances we have discovered appear to have analogues in plants. Ketamine and PCP were the exceptions to this rule, perceived as "highly artificial" compounds. The discovery that ibogaine acts as a PCP/ketamine analog removes this exception, and suggests that the relationship between mind/brain and plant substances is truly comprehensive.

\section{Postscript}

I am no longer as opposed to spiritual explanations of near-death phenomena as my article and this response to the commentaries on it would appear to suggest. Over the past two years (it has been quite some time since I first drafted the article) I have moved more toward the views put forward by John Lilly and Stanislav Grof: namely, that drugs and psychological disciplines such as meditation and yoga my render certain "states" more accessible. The complication then becomes defining just what we mean by "states" and where they are located, if indeed location is an appropriate term at all. But the apparent emphasis on matter over nonmatter contained within this article no longer accurately represents my attitudes.

My forthcoming book Ketamine will consider mystical issues from quite a different perspective, and will give a much stronger voice to those who see drugs as just another door to a space, and not as actually producing that space. After 12 years of studying ketamine, I now believe that there most definitely is a soul that is independent of experience. It exists when we begin, and may persist when we 
end. Ketamine is a door to a place we cannot normally get to; it is definitely not evidence that such a place does not exist.

I regret my past emphasis on the overriding importance of theories of matter as they stood in the late 1980s. Ketamine suggests that we still have much to learn.

\section{References}

Einstein, A. (1935). The world as I see it. London, England: Watts.

Faithfull, M., and Dalton, D. (1994) Faithfull. New York, NY: Penguin, 1994.

Grinspoon, L., and Bakalar, J. B. (1979). Psychedelic drugs reconsidered. New York, NY: Basic Books.

Kales, A. (1987). Sleep disorders: Insomnia, sleepwalking, night terrors, nightmares, and enuresis. Annals of Internal Medicine, 106, 582-592.

Krupitsky, E. M., Grinenko, A. Ya., Berkaliev, T. N., Paley, A. I., Tetrov, U. N., Mushkov, $\mathrm{K}$ A., and Borodkin, Yu. S. (1992). The combination of psychedelic and aversive approaches in alcoholism treatment: The Affective Contra-Attribution Method. Alcoholism Theatment Quarterly, 9(1), 99-105.

Krupitsky, E. M., Grinenko, A. Ya., Karandashova, G. F., Berkaliev, T. N., Moshkov, K. A., and Borodkin, Yu. S. (1990). Metabolism of biogenic amines induced by alcoholism narcopsychotherapy with ketamine administration. Biogenic Amines, 7, 577-582.

Krupitsky, E. M., Paley, A. I., Berkaliev, T. N., Ivanov, V. B., Dubrovina, O. O., Kohanzarova, D. A., Dunaevsky, I. V., Rhizankova, E. B. and Grinenko, A. Ya. (1993). Ketamine-assisted psychedelic therapy. International Journal of Transpersonal Studies, $12,56-60$.

Krystal, J. H., Karper, L. P., Seibyl, J. P., Freeman, G. K, Delaney, R., Bremner, J. D., Heninger, G. R., Bowers, M. B., and Charney, D. S. (1994). Subanesthetic effects of the noncompetitive NMDA antagonist, ketamine, in humans. Archives of General Psychiatry, 51, 199-214.

Lask, B. (1988). Novel and non-toxic treatments for night terrors. British Medical Journal, 297, 592.

Leary, T., Metzner, R. and Alpert, R. (1964). The psychedelic experience: A manual based on The Tibetan Book of the Dead. New York, NY: University Books.

Lilly, J. C. (1978). The scientist: A novel autobiography. New York, NY: Bantam/Lippincott.

Moore, M., and Alltounian, H. (1978). Journeys into the bright world. Rockport, MA: Para Research.

Rogo, D. S. (1984). Ketamine and the near-death experience. Anabiosis: The Journal of Near-Death Studies, 4, 87-96.

Vollenweider, F. X. (1994). Evidence for a cortical-subcortical imbalance of sensory information processing during altered states of consciousness using positron emission tomography and $\left[{ }^{18} \mathrm{~F}\right]$ flourodeoxyglucose. In A. Pletsche and D. Ladewig (Eds.), 50 years of LSD: Current status and perspectives of hallucinogens. A symposium of the Swiss Academy of Medical Sciences (pp. 67-86). London, England: Parthenon.

Vollenweider, F. X. (1996). Relationship of altered states of consciousness and principle components of brain energy metabolism by FDG-PET. Welten des Bewusstseins/Worlds of consciousness: Abstracts of the 2nd International Congress of the European College for the Study of Consciousness, 6, 29-30.

World Health Organization. (1992). The ICD-10 classification of mental and behavioral disorders. Geneva, Switzerland: World Health Organization. 